\title{
New insights into the nature of the Universe obtained
}

\author{
F. N. C. Anyaegbunam (Ph.D.) \\ Department of Physics/Geology/Geophysics Federal University, Ndufu-Alike, Ikwo, Abakaliki. Ebonyi State.
}

\begin{abstract}
Scientists have, in the last few decades, discovered that there is a lot more to the universe than meets the eye: the cosmos appears to be filled with not just one, but two invisible constituents -- dark matter and dark energy -- whose existence has been proposed based solely on their gravitational effects on ordinary matter and energy. In this regard, astrophysicists had postulated the existence of invisible particles called dark matter in order to explain the peculiar motion of galaxies. Based on these observations, they estimate that there must be about 10 times as much (invisible) dark matter in the universe as (visible) ordinary matter. Again dark energy, which produces a repulsive force that appears to be ripping the universe apart was postulated in the 1990's. Scientists invoked dark energy to explain observations that the rate at which the universe appears to be expanding is not slowing, as most cosmologists had thought, but is accelerating instead. It is estimated that three quarters of the universe is made up of dark energy while dark matter accounts for another $23 \%$ and baryonic or ordinary matter and energy occupy only 2\%.Therefore dark energy is hardly science fiction and recent space mission has further proved its existence.
\end{abstract}

Keywords: dark energy, dark matter, baryonic matter, universe.

\section{Introduction}

Astronomy is the window through which scientists can learn about the Universe -- what it is made of; how old it is; and what its future might be. Nevertheless, each culture has its own stories about the sky and the heavenly stars. In ancient China for example, people generally believed that the sky or the "heaven" was important but mythical. Although, there were serious and detailed observational records of the sky yet there were no significant models for predictions about the future behaviour of the Universe. In contrast, around 600 B.C., Greek philosopher Thales of Miletus had already proposed that the Universe is rational and thus could be understood by humans. Around 400 B.C., Plato claimed that "heaven" (meaning the sky) is perfect and the circle is the most perfect form. Thus, the "heaven" is in uniform circular motion with the Earth at the centre. This is the beginning of the geocentric model. But the simple geocentric model cannot explain the retrograde or contrary motion of the planets relative to the Earth's motion round the Sun.. Around 140 A.D. however, an African, Ptolemy (Egyptian), proposed his refined geocentric model. In the Ptolemaic universe, a planet moves in a small circle called an epicycle, and the centre of the epicycle itself moves along a larger circle around the Earth. The centres of the epicycles of the planets Mercury and Venus were supposed to lie on the line joining the Earth and the Sun, while stars are fixed on an outermost sphere. This model gives predictions on the positions of the planets in the sky within a few degrees from the actual positions. This was generally accepted and the Ptolemaic model dominated the western world for about 1500 years.

Then came Nicolaus Copernicus (1473-1543) who proposed the heliocentric model: in which the centre of the universe is the Sun, not the Earth. The Earth is just another planet orbiting around the Sun and it no longer has a special status (the centre) in the Universe. This model is simple and elegant and it predicts the retrograde motion of planets. However the prediction of this model is about as good as the Ptolemaic, though the Copernican model was not commonly accepted at that time. Galileo was a great defender of the heliocentric model. He was the first person who used a telescope to observe the sky. This was the first time that a person used an instrument to enhance one's observing capability. A simplified version of his conflict with the Vatican is that Galileo searched for the truth by observing and doing experiments, but the Vatican believed the truth could only be found in faith. The Inquisition sentenced Galileo to life imprisonment and he was confined in his villa for the last ten years of his life.

\section{Modern Astronomy}

Modern astronomers have built on Galileo's legacy of scientific observations of the Universe using instruments such that today the Universe is understood as being made up of matter and vacuum - at least that is a widely held and apparently banal view. So why look any further? Because all the observations gathered, first by astronomers, then by astrophysicists and, finally, by particle physicists, reflect a universe that is certainly richer and more complex than was first believed.

Einstein's General Theory of Relativity tells us that the future of the Universe, as well as its past, depend on the total quantity of matter and energy contained therein. Observations made so far conclude that we 
must postulate the existence of something called "dark" matter - called dark because it is invisible with the eye or with the aid of any instrument since it emits no light or other detectable radiation. Furthermore, the study of distant supernovas or exploded massive old stars has forced cosmologists to admit the existence of a form of energy related neither to matter nor radiation. As this mysterious energy is very hard to reveal, it has also been dubbed quite simply as "dark energy" -- which is quite different from dark matter..

But the bottom line is that You, the newspaper in your hand, the air we breathe, and the distant stars and galaxies are all made up of elementary particles which physicists call protons, neutrons and electrons. Protons and neutrons are bound together into atomic nuclei while atoms are nuclei surrounded by a full complement of electrons. Hydrogen is composed of one proton and one electron, just as the inert gas helium is composed of two protons, two neutrons and two electrons. Carbon is composed of six protons, six neutrons and six electrons. Heavier elements, such as iron, lead and uranium, contain even larger numbers of protons, neutrons and electrons. Astronomers and physicists like to call all material made up of protons, neutrons and electrons "baryonic matter". Indeed until about twenty years ago, astronomers thought that the universe was composed almost entirely of this "baryonic matter" -- ordinary stuff.

The observable universe is about 46 billion light years in radius.[1] Scientific observation of the Universe has led to inferences of its earlier stages. These observations suggest that the Universe has been governed by the same physical laws and constants throughout most of its extent and history. The Big Bang theory is the prevailing cosmological model that describes the early development of the Universe, which is calculated to have begun $13.798 \pm 0.037$ billion years ago.[2][3] Observations of a supernovae have shown that the Universe is expanding at an accelerating rate, [4].

\section{New Insights}

In the last few decades, scientists have discovered that there is a lot more to the universe than meets the eye: the cosmos appears to be filled with not just one, but two invisible constituents -- dark matter and dark energy -- whose existence has been proposed based solely on their gravitational effects on ordinary matter and energy. In this regard, astrophysicists had postulated the existence of invisible particles called dark matter in order to explain the peculiar motion of galaxies. Based on these observations, they estimate that there must be about 10 times as much (invisible) dark matter in the universe as (visible) ordinary matter. One possible explanation for dark matter is that it is made up of a new type of particle dubbed Weakly Interacting Massive Particles, or WIMPs that don't emit light and barely interact with ordinary matter. As if that weren't enough, in the 1990's along came dark energy, which produces a repulsive force that appears to be ripping the universe apart. Scientists invoked dark energy to explain observations that the rate at which the universe appears to be expanding is not slowing, as most cosmologists had thought, but is accelerating instead. According to the latest estimates, dark energy makes up $\mathbf{7 5}$ per-cent of the universe and dark matter accounts for another $\mathbf{2 3}$ per-cent, leaving baryonic or ordinary matter and energy with a distinctly minority role of only 2 percent [5]. Thus dark energy is hardly science fiction, although no less intriguing and full of mystery for being real science.

Dark energy is today best known as the putative agent of cosmic acceleration -- an unidentified substance that exerts a kind of antigravity force on the universe as a whole. Less well known is that dark energy also has secondary effects on material within the universe. It helped to imprint the characteristic filigree or honey comb distribution of matter on large scales. On a smaller scale, it appears to have choked off the growth of clusters of galaxies some six billion years ago. On a still smaller scale, dark energy has reduced the rate at which galaxies yank on, bang into and merge with one another. Such interactions shape galaxies. Had dark energy been weaker or stronger, the Milky Way might have had a lower star formation rate and our Sun and the heavy elements that constitute our planet might never have been synthesized, and we humans may not have come into being yet. Thus dark energy does more than hurry along the expansion of the universe. It also has a stranglehold on the shape and spacing of galaxies.

Although back in 1998 astronomers became aware that we had been missing nearly three quarters of the contents of the universe, the so-called dark energy -- an unknown form of energy that surrounds each of us, tugging at us ever so slightly, holding the fate of the cosmos in its grip, but to which we are almost very blind. Some researchers, to be sure, had anticipated that such energy existed, but even they will tell you that its detection ranks among the most revolutionary discoveries in 20th-century cosmology. Not only does dark energy appear to make up the bulk of the universe, but also its existence, if it stands the test of time, will probably require the development of new theories of physics. 


\section{Discussions and Conclusion}

Scientists are just starting the long process of figuring out what dark energy is and what its implications are. One realization has already sunk in: although dark energy betrayed its existence through its effect on the universe as a whole, it may also shape the evolution of the universe's inhabitants--stars, galaxies, black holes, galaxy clusters. Astronomers may have been staring at its handiwork for decades without realizing it. Ironically, the very pervasiveness of dark energy is what made it so hard to recognize. Dark energy, unlike matter, does not clump in some places more than others do; by its very nature, it is spread smoothly everywhere. Whatever the location--be it in your kitchen or in intergalactic space--it has the same density, equivalent to a handful of hydrogen atoms per cubic meter. All the dark energy in our solar system amounts to the mass of a small asteroid [which is much smaller than our planet], making it an utterly inconsequential player in the dance of the planets. Its effects stand out only when viewed over vast distances and spans of time.

Since the days of American astronomer Edwin Hubble, observers have known that all but the nearest galaxies are moving away from us at a rapid rate. This rate is proportional to distance: the more distant a galaxy is, the faster its recession. Such a pattern implied that galaxies are not moving through space in the conventional sense of motion but are being carried along as the fabric of space itself stretches. For decades, astronomers struggled to answer the obvious follow-up question: How does the expansion rate change over time? They reasoned that it should be slowing down, as the inward gravitational attraction exerted by galaxies on one another should have counteracted the outward expansion.

The first clear observational evidence for changes in the expansion rate involved distant supernovae, massive exploding stars that can be used as markers of cosmic expansion, just as watching driftwood lets you measure the speed of a river. These observations made clear that the expansion was slower in the past than today and is therefore accelerating. More specifically, it had been slowing down but at some point underwent a transition and began speeding up. This striking result has since been cross-checked by independent studies of the cosmic microwave background radiation by, for example, the Wilkinson Microwave Anisotropy Probe (WMAP).

One possible conclusion is that different laws of gravity apply on super galactic dimensions than on lesser ones, so that galaxies' gravity does not, in fact, resist expansion. But the more generally accepted hypothesis is that the laws of gravity are universal and that some form of energy, previously unknown to science, opposes and overwhelms galaxies' mutual attraction, pushing them apart ever faster. Although dark energy is inconsequential within our galaxy (let alone your kitchen), it adds up to the most powerful force in the cosmos.

As astronomers have explored this new phenomenon, they have found that, in addition to determining the overall expansion rate of the universe, dark energy has long-term consequences for smaller scales. As you zoom in from the entire observable universe, the first thing you notice is that matter on cosmic scales is distributed in a cobweb like pattern--a filigree of filaments like a loose mass of spaghetti, several tens of millions of light-years long, interspersed with voids of similar size. Simulations show that both matter and dark energy are needed to explain this pattern.

Detailed studies indicate that a galaxy gets bent out of shape when it merges with another galaxy in a galactic marriage! The earliest galaxies we can see existed when the universe was about a billion years old, and many of these indeed appear to be merging. As time went on, though, the fusion of massive galaxies became less common. Between two billion and six billion years after the big bang -- that is, over the first half of cosmic history -- the fraction of massive galaxies undergoing a merger dropped from half to nearly nothing at all. Since then, the distribution of galaxy shapes has been frozen, an indication that smash-ups and mergers have become relatively uncommon.

This is a good story, but is it true? Galaxy mergers, black hole activity and star formation all decline with time, and very likely they are related in some way. But astronomers have yet to follow the full sequence of events. On-going surveys with the Hubble Space Telescope, the Chandra X-ray Observatory and sensitive ground-based imaging and spectroscopy will scrutinize these links in coming years.

However, On 21 March 2013, the European research team behind the Planck cosmology probe released the mission's all-sky map of the cosmic microwave background.[7][8][9][10][11] The map suggests the universe is slightly older than thought. According to the map, subtle fluctuations in temperature were imprinted on the deep sky when the cosmos was about 370,000 years old. The imprint reflects ripples that arose as early, in the existence of the universe, as the first nonillionth $\left(10^{-30}\right)$ of a second. Apparently, these ripples gave rise to the present vast cosmic web of galaxy clusters and dark matter. According to the team, the universe is $13.798 \pm$ 0.037 billion years old,[4][12] and contains $4.9 \%$ ordinary matter, $26.8 \%$ dark matter and $68.3 \%$ dark energy. Also, the Hubble constant was measured to be $67.80 \pm 0.77(\mathrm{~km} / \mathrm{s}) / \mathrm{Mpc}$.[7][8][9][11][12] .

An earlier interpretation of astronomical observations indicated that the age of the Universe was 13.772 \pm 0.059 billion years,[13] and that the diameter of the observable universe is at least 93 billion light years or $8.80 \times 10^{26}$ meters,[14]. According to general relativity, space can expand faster than the speed of light, although we can view only a small portion of the Universe due to the limitation imposed by light speed. Since we cannot 
observe space beyond the limitations of light (or any electromagnetic radiation), it is uncertain whether the size of the Universe is finite or infinite.

One way to further investigate the current situation with regard to galactic marriage is to obtain a good census of distant active galaxies and to determine the time when those galaxies last underwent a merger. The analysis will require the development of new theoretical tools but should be within our grasp in the next few years.

\section{References}

[1] Itzhak Bars; John Terning (November 2009). Extra Dimensions in Space and Time. Springer. pp. 27-.ISBN 978-0-387-776378.Retrieved 2011-05-01.

[2] "Planck reveals an almost perfect universe". Planck. ESA. 2013-03-21. Retrieved 2013-03-21.

[3] Planck collaboration (2013). "Planck 2013 results. XVI. Cosmological parameters". Submitted to Astronomy \& Astrophysics.arXiv:1303.5076.Bibcode:2013arXiv1303.5076P.

[4] http://www.nobelprize.org/nobel_prizes/physics/laureates/2011/

[5] Okoye S.E. (2005), Astronomers gain new insight into the nature of the Universe. Short communications.

[6] Universe, ed. Martin Rees, pp. 54-55, Dorling Kindersley Publishing, New York 2005,ISBN 978-0-7566-1364-8

[7] "Planck Reveals An Almost Perfect Universe". ESA. 21 March 2013. Retrieved 2013-03-21.

[8] Clavin, Whitney; Harrington, J.D. (21 March 2013). "Planck Mission Brings Universe Into Sharp Focus". NASA. Retrieved 201303-21.

[9] Overbye, Dennis (21 March 2013). "An Infant Universe, Born Before We Knew". New York Times. Retrieved 2013-03-21.

[10] "Mapping the Early Universe". New York Times. 21 March 2013. Retrieved 2013-03-23.

[11] Boyle, Alan (21 March 2013). "Planck probe's cosmic 'baby picture' revises universe's vital statistics". NBC News. Retrieved 201303-21.

[12] Ade, P. A. R.; Aghanim, N.; Armitage-Caplan, C.; et al. (Planck Collaboration) (20 March 2013). "Planck 2013 results. I. Overview of products and scientific results". Astronomy\& Astrophysics (submitted). arXiv:1303.5062.Bibcode:2013arXiv1303.5062P.

[13] Bennett, C.L.; Larson, L.; Weiland, J.L.; Jarosik, N.; Hinshaw, N.; Odegard, N.; Smith, K.M.; Hill, R.S. et al. (December 20, 2012). Nine-Year Wilkinson Microwave Anisotropy Probe (WMAP) Observations: Final Maps and Results. arXiv:1212.5225.Bibcode:2012arXiv1212.5225B.

[14] Lineweaver, Charles; Davis, Tamara M. (2005). "Misconceptions about the Big Bang". Scientific American. Retrieved 2008-11-06. 